\title{
Internal Energy Deposition in Chemical Ionization/Tandem Mass Spectrometry
}

\author{
Maria P. Ospina, ${ }^{*}$ David H. Powell, and Richard A. Yost \\ Department of Chemistry, University of Florida, Gainesville, Florida, USA
}

\begin{abstract}
The efficiency of the collision-induced dissociation (CID) process as a function of the internal energy deposited into the ion during the ionization event was evaluated. $(\mathrm{M}+\mathrm{H})^{+}$ions of pyrrole, pyrrolidine, pyridine and piperidine (five and six-membered ring heterocyclics) were generated by chemical ionization (CI). The internal energy of the ions was varied by using different reagent gases. Both high-energy $(\mathrm{keV})$ and low-energy $(\mathrm{eV}) \mathrm{CID}$ were performed on these ions. The experiments showed that the $(\mathrm{M}+\mathrm{H})^{+}$ions of the five-membered ring compounds, pyrrole and pyrrolidine, have higher fragmentation efficiencies than the sixmembered ring compounds, pyridine and piperidine. Fragmentation efficiencies in highenergy CID clearly correlate with the internal energy deposited by the ionization technique. Experiments showed that the low-energy CID process is more sensitive than high-energy CID to changes in internal energy. (J Am Soc Mass Spectrom 2003, 14, 102-109) @ 2003 American Society for Mass Spectrometry
\end{abstract}

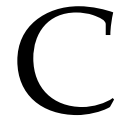
hemical ionization is one of the most widespread ionization techniques used in mass spectrometry. In chemical ionization, collisions between reagent gas ions and sample molecules produce abundant pseudo-molecular ions characteristic of the sample [1]. Ionization in CI can occur by different mechanisms such as charge exchange, electrophilic addition, proton transfer, hydride abstraction, or other anion abstraction mechanism [1-3]. Proton transfer from a reagent gas ion $(\mathrm{B}+\mathrm{H})^{+}$to a sample molecule $\mathrm{M}$ is the most common process and is represented by the equation:

$$
(\mathrm{B}+\mathrm{H})^{+}+\mathrm{M} \rightarrow(\mathrm{M}+\mathrm{H})^{+}+\mathrm{B}
$$

The internal energy $(\Delta \mathrm{H})$ of the $(\mathrm{M}+\mathrm{H})^{+}$ion produced by proton transfer depends on the proton affinities (PA) of the species involved and is described by the equation:

$$
\Delta \mathrm{H}=\mathrm{PA}(\mathrm{B})-\mathrm{PA}(\mathrm{M})
$$

Fragmentation of the $(\mathrm{M}+\mathrm{H})^{+}$ion in CI spectra depends on its internal energy, which can be controlled by varying the reagent gas. In general, fragmentation increases when the exothermicity of the proton transfer reaction increases. It is possible to predict which proton

Published online January 10, 2003

Address reprint requests to Dr. R. A. Yost, Department of Chemistry, University of Florida, P.O. Box 117200, Gainesville, FL 32611-7200, USA. E-mail: ryost@chem.ufl.edu

* Present address: U.S. Centers for Disease Control and Prevention, 4770 Buford Highway NE, Bldg. 17, Mailstop F-17, Atlanta, GA 30341-3724. transfer reactions will occur in a particular system, since values of PA of a large number of compounds are tabulated [4]. CI spectra are often simple, frequently giving only molecular weight information; therefore, activation techniques such as collision with a neutral gas are commonly used to increase the internal energy of the ion and promote fragmentation. It is well known that fragmentation observed in collision-induced dissociation depends upon the internal energy present in the ions prior to collision as well as the energy deposited during collisional activation. There are limited publications on the role of precollisional internal energy on CID spectra and they show conflicting results. Some studies indicate that precursor ion internal energy has a negligible effect on the CID spectrum, except for product ions formed through processes with the lowest activation energy [5-8]. In contrast, other studies indicate that CID spectra can be highly dependent on initial energy of the precursor ion and/or angular momentum [9-12].

The effect of internal energy on the relative rates of the reactions competing in high-energy (HE) CID processes has been studied by varying the electron energy in electron ionization (EI) [5]. According to the results, precursor ion internal energy has a negligible effect on the CID spectrum, except for product ions formed through the processes of lowest activation energy. The effect of the degrees of freedom of the analyte on ion internal energy has also been investigated [9]. In these experiments, the differences in HE-CID spectra of benzoyl ions formed from a series of homologous compounds were attributed to different internal energy distributions in the fragmenting ion. McLafferty et al. [6] reviewed these results by forming the ions by 
electron ionization at 14 and $70 \mathrm{eV}$. They concluded that the HE-CID spectra of benzoyl ions were independent of their internal energy and mode of formation since no differences were observed. An ingenious experiment was designed by Beynon's group [10] in which different portions of a metastable peak were sampled. Ions with different internal energies were selected and allowed to undergo CID. It was shown that the location of an ion within the metastable peak envelope correlated with its internal energy. Thus, the ions sampled from the edges of the metastable peak were the least excited and the ions taken from the center were the most excited, as reflected in the HE-CID spectrum. Bowers and coworkers [11] showed that ions generated by selected ionmolecule reactions could have energies that vary over several electron volts, and that the HE-CID spectrum depends on the internal energy of the ions prior to collision. Later studies performed by the same group [12] included charge-stripping reactions and showed that the charge stripping spectra of the ions studied were strongly dependent on internal energy. The most recent studies on the role of precollisional internal energy on HE-CID spectra were reported by Scrivens and coworkers [13]. In these studies, $(\mathrm{M}+\mathrm{H})^{+}$ions from Irganox 1076, a polymer additive were formed by various ionization techniques and then allowed to undergo CID. It was demonstrated that the variation in relative abundance of various fragment ions present in the CID spectra was correlated to differences in the internal energy transferred to the precursor ion during ionization.

Although there are several studies that indicate the importance of internal energy on CID spectra, it is still often assumed that the ions have no memory of where and how they were formed, as implied by the quasiequilibrium theory. We are interested in understanding the effects of internal energy on high- and low-energy (LE) CID spectra. In the present work, $(\mathrm{M}+\mathrm{H})^{+}$ions of several heterocyclic amines were formed with different internal energies via chemical ionization using different reagent gases. The effect of precollisional internal energy on the subsequent CID spectra of these amines is reported.

\section{Experimental}

Pyridine, piperidine, pyrrole, and pyrrolidine (Figure 1) were purchased from Aldrich Chemical Co. (Milwaukee, WI). These compounds were used as received, except for pyrrole, which was passed through a silica gel column to remove oxidation products. The compounds were introduced into the mass spectrometer through a variable leak valve (Granville Phillips, Boulder, CO, Series 203). The valve was placed inside the oven of a gas chromatograph so that its temperature could be regulated as needed. Mass spectra were obtained on a Finnigan MAT95Q (Finnigan MAT, San Jose, CA) hybrid sector mass spectrometer of BEoQ configuration, where o is a RF-only octopole, which acts

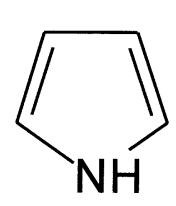

Pyrrole

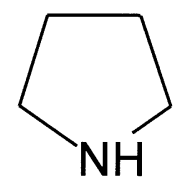

Pyrrolidine

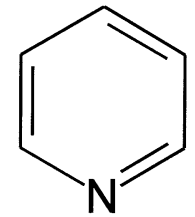

Pyridine

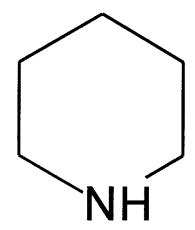

Piperidine
Figure 1. Five- and six-membered heterocyclic compounds.

as a collision cell. The compounds were ionized by positive chemical ionization using methane, isobutane and ammonia as reagent gases, with their pressures adjusted to obtain an $[\mathrm{M}+\mathrm{H}]^{+} / \mathrm{M}^{+}$ratio of $10 \pm 3$. Even though the MAT95Q has separate lines for each of the reagent gases used in the experiments, the gas lines and the ion source were pumped down for thirty min after changing reagent gases in order to minimize cross-contamination of the spectra, as indicated by the absence of reagent ions from the previous reagent gas.

For high-energy CID experiments, $(\mathrm{M}+\mathrm{H})^{+}$ions of interest were selected with the magnetic sector and allowed to collide with helium at $5 \mathrm{keV}$ in the field-free region between the magnet and the electric sector. Helium pressure was increased from $10^{-6}$ to $10^{-5} \mathrm{mbar}$ (measured as analyzer pressure because of the ion gauge position, and uncorrected for $\mathrm{He}$ ) until $97 \%$ or more of the precursor ion intensity was attenuated. The product ions were analyzed according to their kinetic energies by scanning the electric sector. This technique is also known as MIKES [14]. Twenty scans were acquired and averaged at each collision gas pressure. Metastable ions were also studied by MIKES in the absence of collision gas.

Precursor ions for low-energy CID were selected by the double focusing (BE) mass spectrometer, decelerated to the appropriate collision energy and passed to the octopole collision cell where they collided with Argon as the collision gas. Collision gas pressure was increased from $10^{-6}$ to $10^{-5} \mathrm{mbar}$. The collision energy was varied from 5 to $95 \mathrm{eV}$ in $10 \mathrm{eV}$ increments at each collision gas pressure by changing the offset voltage applied to the octopole. Product ions were mass analyzed with the quadrupole mass analyzer of the MAT95Q. Ten scans were acquired and averaged at each collision energy.

High- and low-energy CID experiments were performed in triplicate for each reagent gas and an average of the three analyses was used for all the calculations. 
Table 1. Proton affinity values for species of interest

\begin{tabular}{lc}
\hline Species & $\mathrm{PA}(\mathrm{eV})^{*}$ \\
\hline \hline $\mathrm{C}_{4} \mathrm{H}_{5} \mathrm{~N}$ Pyrrole & 9.07 \\
$\mathrm{C}_{4} \mathrm{H}_{9} \mathrm{~N}$ Pyrrolidine & 9.82 \\
$\mathrm{C}_{5} \mathrm{H}_{5} \mathrm{~N}$ Pyridine & 9.63 \\
$\mathrm{C}_{5} \mathrm{H}_{9} \mathrm{~N}$ Piperidine & 9.89 \\
$\mathrm{CH}_{4}$ Methane & $5.63\left(\mathrm{CH}_{5}\right)^{+}$ \\
& $7.05\left(\mathrm{C}_{2} \mathrm{H}_{5}\right)^{+}$ \\
$\mathrm{C}_{4} \mathrm{H}_{8}$ Isobutane & 8.31 \\
$\mathrm{NH}_{3}$ & 8.84 \\
\hline
\end{tabular}

*Values obtained from reference 4 and converted from $\mathrm{kcal} / \mathrm{mol}$ to $\mathrm{eV}$.

Most of the graphs shown here include error bars equivalent to plus and minus one standard deviation.

\section{Results and Discussion}

Chemical ionization with methane, isobutane and ammonia successfully produced $(\mathrm{M}+\mathrm{H})^{+}$ions that underwent CID. Based on the proton affinities of the species involved and by calculating the exothermicity of the proton transfer reaction with eq 2 , the ionization process with methane $\mathrm{CI}$ (reagent ions $\mathrm{CH}_{5}^{+}$and $\mathrm{C}_{2} \mathrm{H}_{5}^{+}$) is expected to be the most exothermic, and therefore, the $(\mathrm{M}+\mathrm{H})^{+}$generated with methane will have the most internal energy. On the other hand, proton transfer reactions involved in ammonia $\mathrm{CI}$ will be the least exothermic and the $(\mathrm{M}+\mathrm{H})^{+}$ions will have the least internal energy. Proton affinities of the compounds and reagent gases used in these experiments are shown in Table 1.

\section{Metastable Ions}

A metastable ion is one that is sufficiently stable to survive the ionization conditions of the ion source, but is not stable enough to reach the detector before dissociating [15]. Excess internal energy imparted during ionization may be released upon dissociation as translational energy of the dissociation products. This causes a spread in the velocity of the product ions, resulting in broadening of the metastable ion decomposition peak shape. Thus, kinetic energy release (KER) measurements allow precise estimation of a quantity related to the internal energy distribution of the precursor ion [16].

KER [14] values for the metastable decompositions of the $(\mathrm{M}+\mathrm{H})^{+}$ions formed by $\mathrm{CI}$ with different reagent gases were calculated in order to correlate the internal energy deposited by the ionization technique with its effect on tandem mass spectra. The relative intensities of the metastable ions were very low $(<0.03 \%$ of the normalized precursor ion intensity). For the compounds studied, no metastable transitions were observed for $(\mathrm{M}+\mathrm{H})^{+}$ions generated by isobutane or ammonia CI. The absence of metastable ions indicates that the internal energy of the precursor ion prior to collisions must be less than the critical energy for the pathway of lowest dissociation energy [15]. For protonated pyrrole, a metastable ion at $\mathrm{m} / \mathrm{z} 41$ was observed only when methane was used as reagent gas, which corresponds to the neutral loss of HCN. For pyridine, no metastable ion decomposition is observed for the $(\mathrm{M}+\mathrm{H})^{+}$ion $(\mathrm{m} / \mathrm{z} 80)$ even with methane $\mathrm{CI}$, which imparts $4 \mathrm{eV}$ of internal energy. A metastable ion at $\mathrm{m} / \mathrm{z}$ 30 was observed for pyrrolidine with methane and isobutane CI produced ions. Two metastable ions, $\mathrm{m} / \mathrm{z}$ 69 and $m / z$ 30, which correspond to neutral losses of 17 $\mathrm{u}\left(\mathrm{NH}_{3}\right)$ and $56 \mathrm{u}\left(\mathrm{C}_{3} \mathrm{H}_{6} \mathrm{~N}\right)$, respectively were observed for piperidine $(\mathrm{M}+\mathrm{H})^{+}$ion generated by methane $\mathrm{CI}$. The peak at $m / z 30$ in the metastable ion spectra of piperidine and pyrrolidine is common for secondary amines and corresponds to $\mathrm{NH}_{2} \mathrm{CH}_{2}^{+}$produced by rearrangement [17].

Correlation of internal energy deposited by proton transfer reactions from various reagent gases with metastable kinetic energy release was not possible due to the lack of metastable ions with reagent gases other than methane. The low intensity of the metastable ions and associated large error bars for methane CI preclude any correlation of PA and KER for the molecules studied.

\section{Fragmentation Efficiency/HE-CID}

Fragmentation efficiency $\left(E_{f}\right)$ is defined as the fraction of detected ions that are fragment ions [18] and is given by

$$
E_{f}=\left(\frac{\Sigma F_{i}}{\Sigma F_{i}+P}\right)
$$

where $\Sigma F_{i}$ is the sum of the intensities of all the fragment ions, $P$ is the remaining precursor ion intensity and $P_{o}$ is defined as the parent ion intensity when no collision gas is introduced. In order to estimate the energy deposited by the ionization technique and its effect on HE-CID the fragmentation efficiencies for the $(\mathrm{M}+\mathrm{H})^{+}$ions formed by $\mathrm{CI}$ with the different reagent gases were compared. In these studies, fragmentation efficiency was calculated as the collision gas pressure was increased. Because the pressure of the collision gas is typically not measured directly in the collision cell of a sector mass spectrometer but rather at a nearby location, it has been suggested that precursor ion transmission $\left(P / P_{o}\right)$ or attenuation $\left(1-P / P_{o}\right)$ be measured instead of pressure $[18,19]$. Although measuring attenuation takes care of differences in both collision cell designs and pressure gauges, and facilitates comparison of data taken with different instruments, $[18,19]$ it will be shown that pressure is a more useful parameter to plot.

Increasing the collision gas pressure increases the fragmentation efficiency; the collection efficiency, however, decreases as the collision gas pressure increases because of scattering and neutralization losses in the 

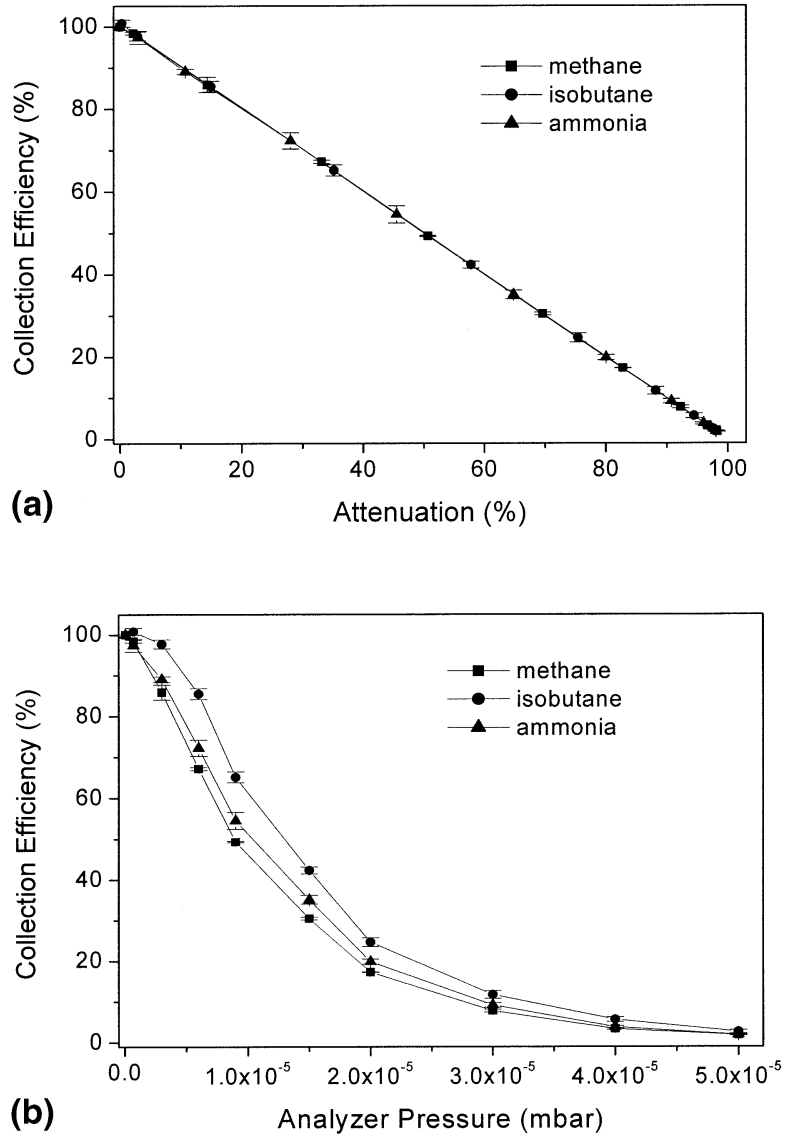

Figure 2. Collection efficiency curves for the HE-CID of $(\mathrm{M}+$ $\mathrm{H})^{+}$ions of piperidine produced by CI with methane, isobutane, and ammonia as a function of: (a) Attenuation; (b) analyzer pressure.

field free region used for CID. The collection efficiency $\left(E_{c}\right)$ represents the ratio of the intensity of the ions leaving the collision region that are collected to the intensity of precursor ions in the absence of collision gas [18] and is given by the equation:

$$
E_{c}=\left(\frac{\Sigma F_{i}+P}{P_{o}}\right)
$$

Figure 2 compares the collection efficiency of piperidine $(\mathrm{M}+\mathrm{H})^{+}$ions as a function of attenuation and collision gas pressure (measured as analyzer pressure) and chemical ionization reagent gas. In both cases, the collection efficiency of the ions formed by CI with methane, isobutane and ammonia is calculated at $100 \%$ when there is no collision gas present. For collection efficiencies measured as a function of attenuation, the collection efficiencies decrease linearly and are virtually indistinguishable (Figure 2a) from one another when beam attenuation increases because of scattering and neutralization reactions of the ions occurring in the collision region. For collection efficiencies evaluated as a function of pressure in the analyzer (Figure $2 b$ ), slight differences are observed at a given pressure. Thus, the

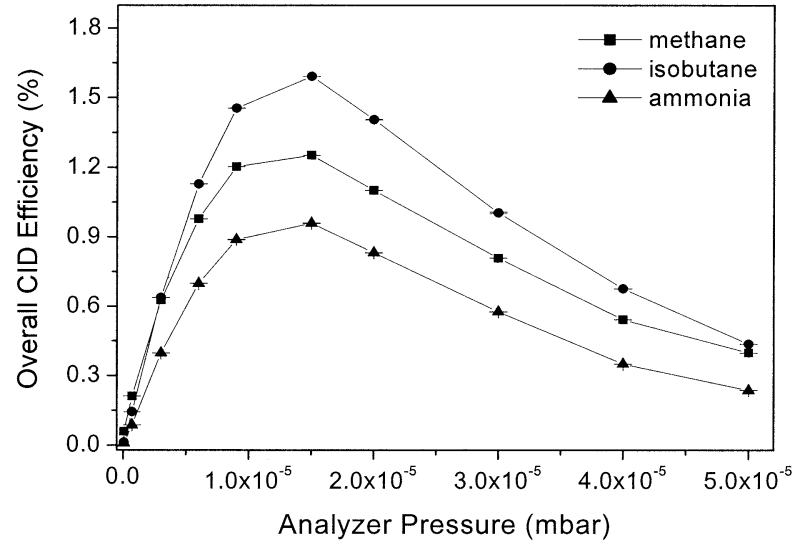

Figure 3. Overall CID efficiency curves for the HE-CID of (M + $\mathrm{H})^{+}$ions of piperidine produced by CI with methane, isobutane, and ammonia as a function of analyzer pressure.

collection efficiency is highest for ions formed by isobutane $\mathrm{CI}$, followed by the collection efficiency of ions formed by ammonia $\mathrm{CI}$, in turn followed by those generated by methane. Since all experiments were done in the same instrument and the use of attenuation hides the true nature of the collection efficiency pressure will be used in all graphs from now on.

As was mentioned before, as fragmentation efficiency increases, the collection efficiency decreases. The product of these two values is called the overall CID efficiency $\left(E_{C I D}\right)$ and accounts for fragmentation, neutralization and scattering losses during the CID process. The utility of plotting overall CID efficiency, however, is that it provides a much better way to view differences in efficiencies at low attenuations than does plotting fragmentation efficiencies. The overall CID efficiency has been defined as the fraction of initial precursor ion that is converted to collected product ions or the total fragment ion current divided by the intensity of the precursor ion in the absence of collision gas [18] and can be written as:

$$
E_{C I D}=\frac{\Sigma F_{i}}{P_{o}}=E_{f} \times E_{c}
$$

The overall CID efficiency plots for $(\mathrm{M}+\mathrm{H})^{+}$ions of piperidine generated by CI with methane, isobutane and ammonia as a function of analyzer pressure are shown in Figure 3. As collision gas pressure is increased, overall CID efficiency curves typically show an increase and level off at the higher pressures. A decrease is observed at high pressures due mostly to scattering losses. By evaluating the overall efficiency of the HE-CID process as a function of either attenuation or pressure, the highest values are obtained for ions generated by isobutane $\mathrm{CI}$, followed by those of methane CI. The lowest overall efficiencies are observed for ions produced by ammonia CI. Note that the high values of the collection efficiency for isobutane CI 

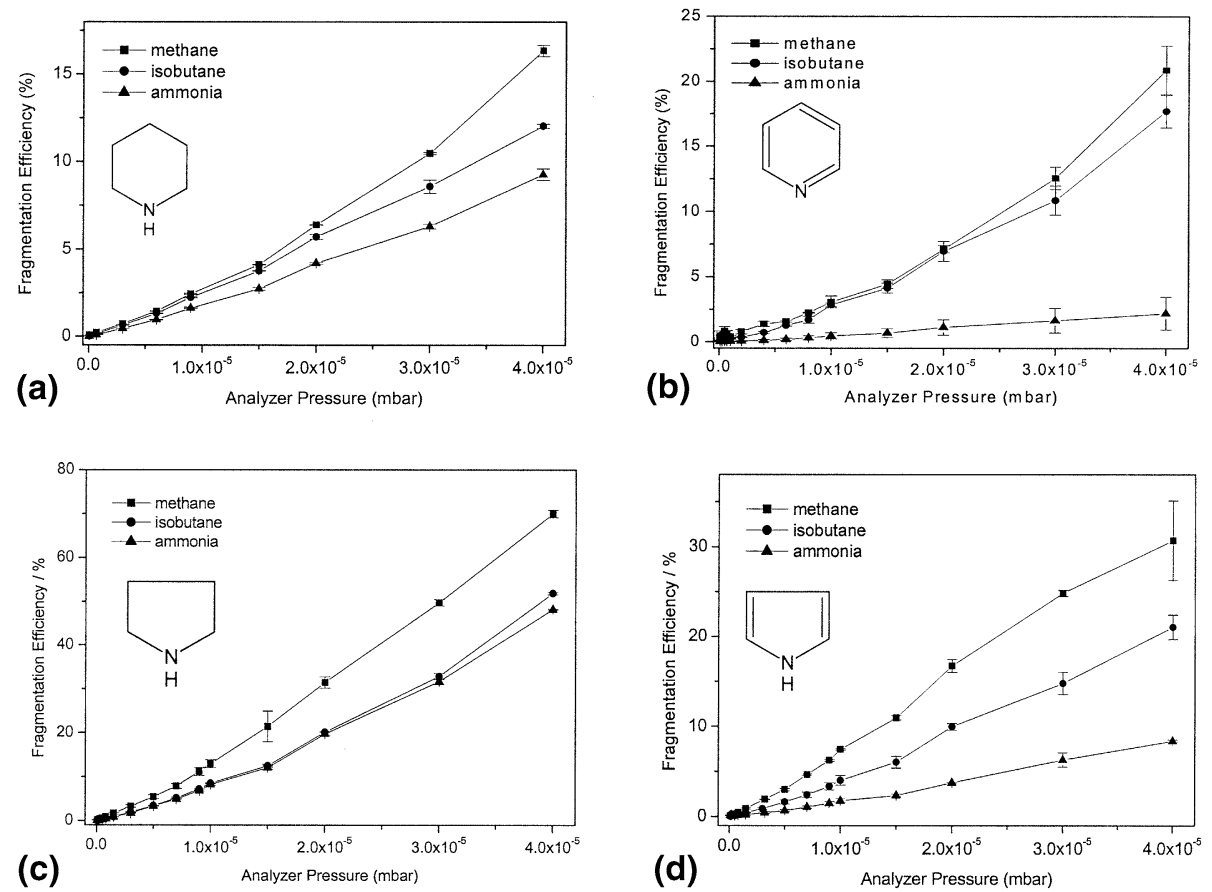

Figure 4. The effect of collision gas pressure (measured as analyzer pressure) and CI reagent gas on fragmentation efficiency of $(\mathrm{M}+\mathrm{H})^{+}$ions from: (a) Piperidine; (b pyridine; (c) pyrrolidine; (d) pyrrole.

generated ions are amplified in these graphs.

The primary goal of this study is to evaluate the effect of internal energy on the fragmentation process. Thus, it is most informative to examine the fragmentation efficiency curves rather than the collection or overall efficiency curves, both of which are affected by scattering and neutralization. Furthermore, it is preferable to plot fragmentation efficiency versus analyzer pressure rather than attenuation, since attenuation is also affected by scattering and neutralization losses.

The dependence of HE-CID fragmentation efficiency on the collision gas pressure (plotted as pressure measured directly in the analyzer region) and on the reagent gas used for chemical ionization is shown in Figure 4 for the $(\mathrm{M}+\mathrm{H})^{+}$ions of piperidine, pyridine, pyrrolidine, and pyrrole. As anticipated, fragmentation efficiency increases linearly as collision gas pressure is increased and the internal energy of the $(\mathrm{M}+\mathrm{H})^{+}$ions increases more rapidly as the number of collisions increases, resulting in more fragmentation. In all cases, the fragmentation degree of the $(\mathrm{M}+\mathrm{H})^{+}$ions during CID is dependent upon the CI reagent gas used. Fragmentation efficiency is the greatest for methane as reagent gas, least for ammonia, and intermediate for isobutane. This agrees with larger internal energy deposited into the $(\mathrm{M}+\mathrm{H})^{+}$ions by $\mathrm{CI}$ with reagent gases of lower proton affinities. Notice that the fragmentation efficiencies at any given gas pressure of the five-membered heterocycles pyrrolidine and pyrrole (Figure 4c and $d$, respectively) are much higher than those of the six-membered compounds piperidine and pyridine (Figure $4 a$ and $b$, respectively). Such internal energy effects are less evident in the bigger species (higher degrees of freedom) because the energy is distributed among more internal modes. This also agrees with the study of metastable ions in which the five-membered ring compounds showed more fragments than the six-membered rings. Moreover, for the heteroaromatic compounds, because of the excessive electron density ( $\pi$-excessive system) in pyrrole, electrophilic addition such as $\mathrm{H}^{+}$addition should be more readily accomplished than in pyridine, which is considered a $\pi$-deficient system [20]. Even under multiple collision conditions, the fragmentation efficiency is still affected by the energy deposited by the ionization technique; therefore, the internal energy differences are not masked by the HE-CID process.

It has been claimed [5] that a unique advantage of CID spectra for structure determination is their virtual insensitivity to ion internal energy, if the peaks resulting from low activation energy pathways are ignored. The CID spectra of precursor ions of the same structure formed with different internal energies will have the same relative abundances within statistical error, if that assumption is valid. The results presented here indicate that fragmentation efficiencies of the heterocyclic compounds are a function of the internal energy deposited during ionization. Note that the fragmentation efficiencies are a function of the total intensity of the product ions in the CID spectra; the relative abundances may or may not be affected.

To get a better understanding of the effect of internal energy on relative abundance of product ions, the intensity ratios of several product ions in the HE-CID 

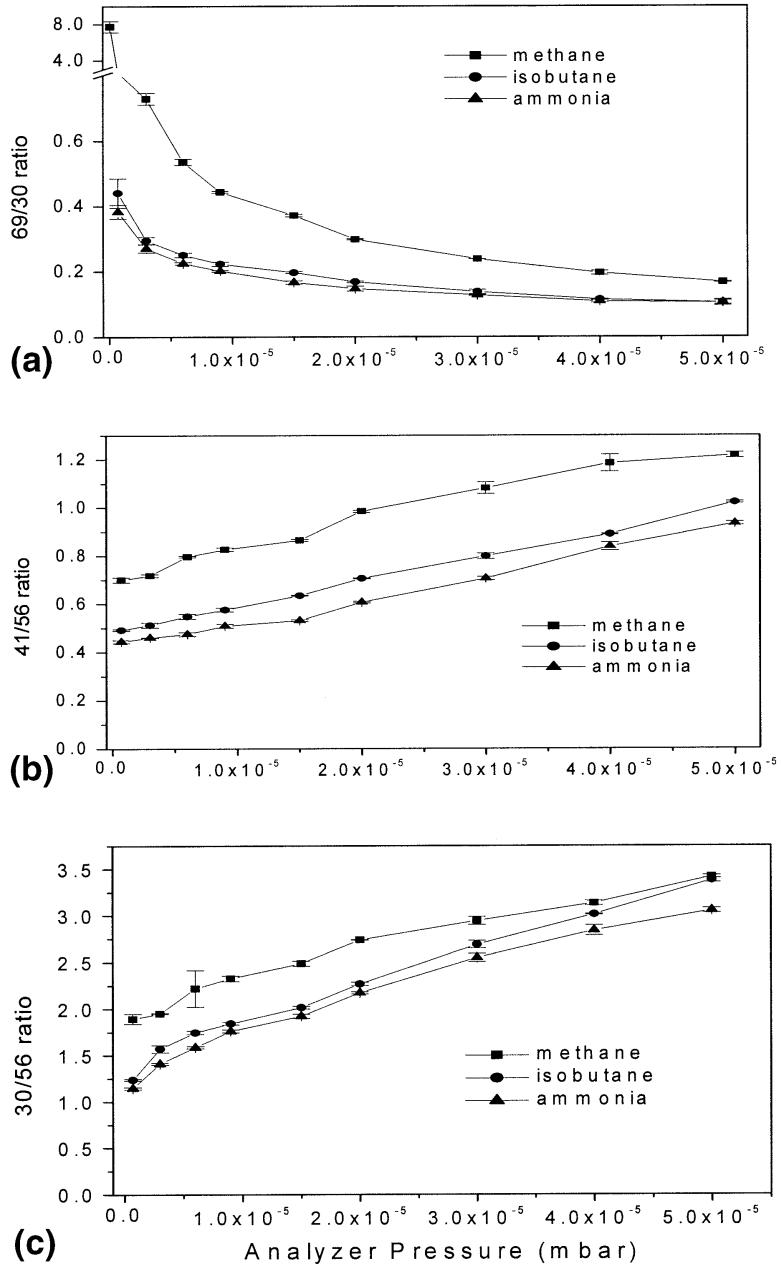

Figure 5. The effect of collision gas pressure (measured as analyzer pressure) and $\mathrm{CI}$ reagent gas on several fragment ion ratios from protonated piperidine. HE-CID $5 \mathrm{keV}$, helium used as collision gas. $\mathrm{m} / \mathrm{z} 30$ and 69 are metastable ions.

spectrum of $(\mathrm{M}+\mathrm{H})^{+}$ions of piperidine were plotted as a function of collision gas pressure and CI reagent gas. For each ion and at each pressure, ten scans were averaged and the relative intensity of the fragment ions plotted. Figure 5 shows the effect of varying the collision gas pressure and the CI reagent gas on several ion ratios from HE-CID spectra of protonated piperidine. The ions used for these ratios range from metastable ions $[\mathrm{m} / \mathrm{z} 69$ and 30, which correspond to the neutral losses of $17 \mathrm{u}\left(\mathrm{NH}_{3}\right)$ and $56 \mathrm{u}\left(\mathrm{C}_{3} \mathrm{H}_{6} \mathrm{~N}\right)$, respectively] to ions formed purely during $\operatorname{CID}(\mathrm{m} / \mathrm{z} 41,56)$. In all cases, at a given collision gas pressure the ion ratios are higher for ions formed by HE-CID of the $(\mathrm{M}+\mathrm{H})^{+}$ions produced by methane $\mathrm{CI}$, followed by those of the ions formed by isobutane CI, in turn followed by those of the ions formed by ammonia CI. Recall that $\mathrm{m} / \mathrm{z} 69$ and 30 are metastable ions from $(\mathrm{M}+\mathrm{H})^{+}$of piperidine, and thus are formed by low activation energy pathways. Notice that the $69 / 30$ ion ratio with methane CI is 2 to 20 times higher than for isobutane or ammonia CI at the same pressure. Clearly, internal energy has an enormous effect on this ratio. As collision gas pressure is increased, the $69 / 30$ ratios get to a point where they level off and remain nearly constant. The behavior observed for the metastable ions was expected, since ion internal energies have been reported to have an effect on relative abundances of ions of low activation energies such as the metastable ions.

Ion ratios were also evaluated for ions that are formed only from CID processes ( $m / z 41$ and 56) and from a combination of metastable and CID processes $(\mathrm{m} / \mathrm{z} 30$ and 56). For the graphs considering 41/56 and $30 / 56$ ratios, and within the experimental error, there is a clear effect of the precollisional internal energy on the product ion ratios, as given by the order of the curves. In these graphs, methane is always the top curve, followed by isobutane, in turn followed by the curve obtained under ammonia CI. In general, even though these are not ions formed via low activation energy pathways, the effect of precollisional internal energy was not masked by the energy deposited by the CID process.

Calculation of ion ratios was also performed for ions resulting from the HE-CID of $(\mathrm{M}+\mathrm{H})^{+}$from pyridine. Metastable ions from pyridine were not observed, even when methane (which imparts approximately $4 \mathrm{eV}$ of internal energy) was used as CI reagent gas. The CID process, in contrast, produced ions at $m / z 27,28,39$, and 52. In Figure 6 the 28/52, 27/52, and 39/52 ion ratios for pyridine are plotted as a function of collision gas pressure and CI reagent gas. Note that at low collision gas pressures no fragment ions were observed. In all cases there is a clear separation among the curves obtained with methane, isobutane and ammonia CI and the curves are always similarly ordered. Indeed, the $28 / 52$ ion ratio is approximately two times higher for pyridine $(\mathrm{M}+\mathrm{H})^{+}$ions produced by methane or isobutane CI than for those produced by ammonia. Clearly, fragment ion ratios are significantly affected by choice of CI reagent gas. This indicates that the effect of precollisional internal energy is still observed under HE-CID conditions, even when the ions are not formed by low activation energy processes.

It is important to mention that for the compounds shown here, the high-energy CID spectra for the $(\mathrm{M}+$ $\mathrm{H})^{+}$ions obtained with the different reagent gases were similar, which indicates that ions with the same structures were produced. The differences in the fragmentation efficiency curves can be attributed to differences in internal energy of the ions previous to CID.

\section{LE-CID versus HE-CID}

The effect of the collision gas pressure and collision energy on the appearance of CID spectra is generally more pronounced at low energies $(<100 \mathrm{eV})$ than at high energies (keV) [21]. Thus, it should be informative to compare the effect of reagent gas identity and collision gas pressure on LE-CID with those on HE-CID.

LE-CID spectra were obtained with argon as collision gas for the $(\mathrm{M}+\mathrm{H})^{+}$ions from pyridine, pyrroli- 

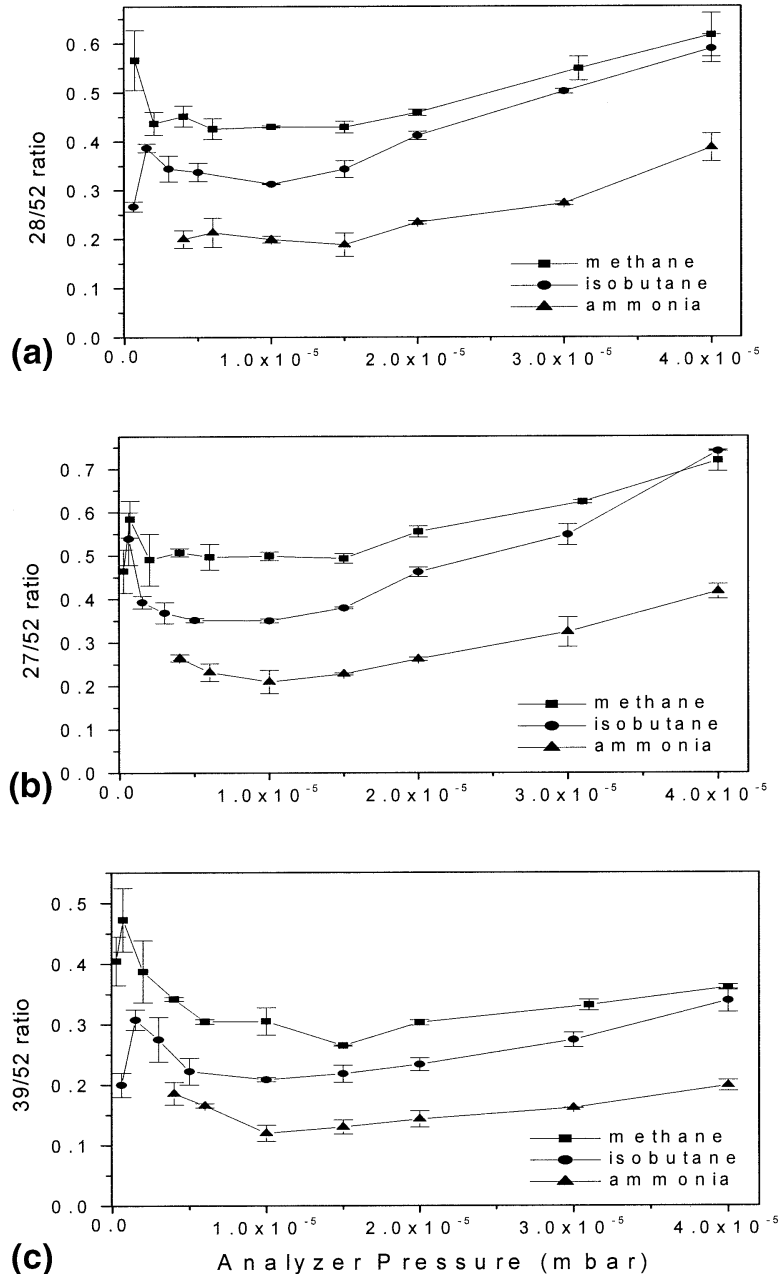

Figure 6. The effect of collision gas pressure (measured as analyzer pressure) and CI reagent gas on several fragment ion ratios from protonated pyridine. HE-CID $5 \mathrm{keV}$, helium used as collision gas.

dine, and pyrrole at different collision energies and collision gas pressures. LE-CID of pyridine showed only one product ion, $\mathrm{m} / \mathrm{z} 53$ and no ion ratios were calculated. LE-CID spectra of piperidine were not obtained because of irreparable problems in the quadrupole section of the hybrid instrument. Thus, the comparison of LE-CID and HE-CID was done only for pyrrolidine and pyrrole.

Decomposition of protonated pyrrolidine by lowenergy CID showed intense ions at $\mathrm{m} / \mathrm{z} 18,30,44$, and 55. Recall that $\mathrm{m} / \mathrm{z} 30$ is a metastable ion in the HE-CID spectrum which corresponds to $\mathrm{NH}_{2} \mathrm{CH}_{2}^{+}$and it is produced by rearrangement [17], and thus must arise from a low activation energy pathway. Ion ratios were calculated between $\mathrm{m} / \mathrm{z} 55$ and 30 at both high and low collision energies. The 55/30 fragment ion ratios in HE-CID for the $(\mathrm{M}+\mathrm{H})^{+}$ions from pyrrolidine are consistent throughout the entire pressure range, with ratios for methane $\mathrm{CI}$ higher than those of isobutane $\mathrm{CI}$, in turn followed by those of ammonia CI. For LE-CID at $65 \mathrm{eV}$, ammonia CI shows the lowest ratios, and meth-

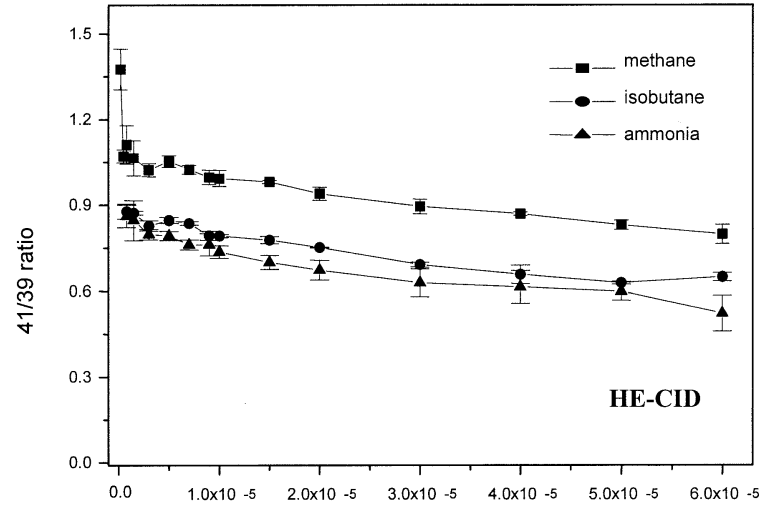

(a)

Analyzer Pressure (mbar)

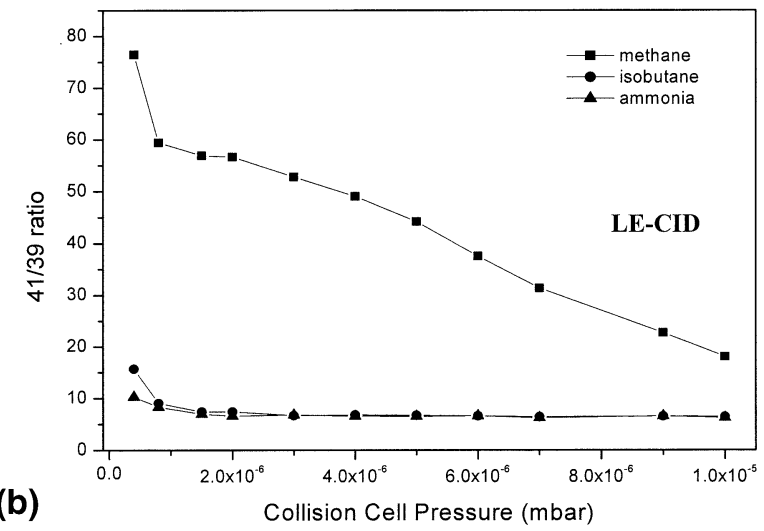

Figure 7. The effect of collision gas pressure (measured directly as analyzer pressure) and CI reagent gas on the 41/39 fragment ion ratio from protonated pyrrole. (a) HE-CID $5 \mathrm{keV}$; (b) LE-CID $65 \mathrm{eV} . \mathrm{m} / \mathrm{z} 41$ is a metastable ion.

ane and isobutane CI curves overlap above $6 \times 10^{-6}$ mbar. Clearly, internal energy imparted during $\mathrm{CI}$ has a significant effect on the decomposition of pyrrolidine by both LE-CID and HE-CID spectra; the differences in internal energy from the ionization technique become less important, however, at high collision gas pressures in LE-CID.

The relative intensities of ions $\mathrm{m} / \mathrm{z} 41$ and 39 formed under high- and low-energy CID of $(\mathrm{M}+\mathrm{H})^{+}$ions of pyrrole are shown in Figure 7. The ion at $\mathrm{m} / \mathrm{z} 41$ corresponds to the neutral loss of $\mathrm{HCN}$ from protonated pyrrole and $\mathrm{m} / \mathrm{z} 39$ corresponds to the subsequent loss of $\mathrm{H}_{2}$. For LE-CID, $m / z 41$ is predominant over $\mathrm{m} / \mathrm{z}$ 39 (i.e., the ratio is much greater than 1 ) in all three $\mathrm{CI}$ experiments. The ratios of intensities for $\mathrm{m} / \mathrm{z} 41$ to 39 range from 75:1 to 6:1. In contrast, for HE-CID, the relative intensity of these two ions is generally about 1:1. This suggests that formation of $\mathrm{m} / \mathrm{z} 39$ ion involves a reaction channel available at higher energies, not only because its relative intensity is considerably higher in HE-CID than in LE-CID, but also because its relative intensity increases at higher collision gas pressures. Studies by Claeys and coworkers [22] on high- and low-energy CID spectra of ricinoleic and ricinelaidic 
acid revealed that the associated loss of hydrogen corresponds to a high-energy CID fragmentation process. Clearly, the relative intensities of the product ions in both high- and low-energy CID are affected by internal energy imparted during ionization. These effects are more pronounced in LE-CID, where the ratio varies by up to a factor of 10 as the reagent gas is changed.

\section{Conclusions}

Previous publications on the role of precollisional internal energy on CID spectra have clearly demonstrated the disagreement about whether or not precursor ion internal energy has a significant effect. Indeed, it is still often assumed that the ions have no memory of where and how they were formed. The data presented here directly address the effects of internal energy on highand low-energy CID spectra, particularly for the $(\mathrm{M}+$ $\mathrm{H})^{+}$ions of several heterocyclic amines formed with different internal energies via chemical ionization using different reagent gases.

The $(\mathrm{M}+\mathrm{H})^{+}$ions of the five-membered heterocyclic compounds, pyrrole and pyrrolidine, have higher fragmentation efficiencies than the six-membered rings, pyridine and piperidine, which agrees with the larger number of ions observed in the metastable ion spectra of the five-membered compounds; therefore, more fragmentation is induced in these systems. As expected from the exothermicity of the proton transfer reaction, $(\mathrm{M}+\mathrm{H})^{+}$ions produced by methane CI showed the highest fragmentation efficiencies, followed by those produced by isobutane CI. The lowest fragmentation efficiencies were observed for ions produced by ammonia CI. Fragmentation efficiencies in HE-CID clearly correlate with the amount of internal energy deposited by the ionization technique for the compounds studied. High-energy CID spectra include more abundant product ions from reaction channels such as hydrogen loss that open up at high energies. For CID of $(\mathrm{M}+\mathrm{H})^{+}$ions of pyrrolidine and pyrrole, the low-energy process is more sensitive to changes in internal energy at low collision cell pressures.

Neutralization-reionization studies and calculations have shown $[23,24]$ that under isobutane and ammonia $\mathrm{CI}$, protonation of pyrrole occurs on the ring carbon atoms rather than on the nitrogen. In contrast, pyridine is protonated exclusively on the nitrogen under isobutane and ammonia $\mathrm{CI}$; under methane $\mathrm{CI}$, it can be protonated on the nitrogen or on the ring carbons. The CID spectra for the $(\mathrm{M}+\mathrm{H})^{+}$ions studied here were so similar that ions with the same structures can be assumed and that the differences in fragmentation efficiencies can be attributed to precollisional internal energy. The difference in protonation sites may play a role in some of the differences observed in our experiments, however, and is important to address in future studies.

\section{References}

1. Harrison, A. G. Chemical Ionization Mass Spectrometry. CRC Press: Boca Raton, 1992, p 27-28.

2. Hunt, D. F., in Tandem Mass Spectrometry, McLafferty, F. W., ed.; Wiley: New York, 1983, pp 106-113.

3. Chapman, J. R. Practical Organic Mass Spectrometry. Wiley: Chichester, 1993 , pp 75-82.

4. NIST Chemistry Webbook, NIST Standard Reference Database; Number 69, March 1998 on-line release, http// webbook.nist.gov/chemistry.

5. McLafferty, F. W.; Bente, P. F.; Kornfeld, R.; Tsai, S. C.; Howe, I. J. Am. Chem. Soc. 1973, 95, 2120-2129.

6. McLafferty, F. W.; Hirota, A.; Barbalas, M. P.; Pegues, R. F. Int. J. Mass Spectrom. Ion Phys. 1980, 35, 299-303.

7. Curtis, J. M.; Bradley, C. D.; Derrick, P. D.; Sheil, M. M. Org. Mass Spectrom. 1992, 27, 502-507.

8. Sheil, M. M.; Kilby, G. W.; Curtis, J. M.; Bradley, C. D.; Derrick, P. D. Org. Mass Spectrom. 1993, 28, 574-576.

9. Porter, C. J.; Morgan, R. P.; Beynon, J. H. Int. J. Mass Spectrom. Ion Phys. 1978, 28, 321-333.

10. Porter, C. J.; Proctor, C. J.; Beynon, J. H. Org. Mass Spectrom. 1981, 16, 62-67.

11. Van Koppen, P. A. M.; Illies, A. J.; Liu, S.; Bowers, M. T. Org. Mass Spectrom. 1982, 17, 399-402.

12. Jarrold, M. F.; Illies, A. J.; Kirchner, N. J.; Bowers, M. T. Org. Mass Spectrom. 1983, 18, 388-395.

13. Jackson, A. T.; Jennings, K. R.; Scrivens, J. H. Rapid Commun. Mass Spectrom. 1996, 10, 1459-1462.

14. Cooks, R. G.; Beynon, J. H.; Caprioli, R. M.; Lester, G. R. Metastable Ions. Elsevier: New York, 1973.

15. Bush, K. L.; Glish, G. L.; McLuckey, S. A. Mass Spectrometry/ Mass Spectrometry. VCH Publishers: New York, 1988, pp 55-60.

16. Brenton, A. G. J. Mass Spectrom. 1995, 30, 657-665.

17. Kitson, F. G.; Larsen, B. S.; McEwen, C. N. Gas Chromatography and Mass Spectrometry. Academic Press: San Diego, 1996, p 80.

18. Yost, R. A.; Fetterolf, D. D. Mass Spectrom. Rev. 1983, 2, 1-45.

19. Hayes, R. N.; Gross, M. L. McCloskey, J. A., Ed. Methods in Enzymology, Vol CXCIII. Academic Press: San Diego, 1990, p 242.

20. Newkome, G. R.; Paulder, W. W. Contemporary Heterocyclic Chemistry. Wiley-Interscience: New York, 1982, p 15.

21. Douglas, D. J. J. Phys. Chem. 1982, 86, 185-191.

22. Nizigiyimana, L.; Van den Heuvel, H.; Claeys, M. J. Mass Spectrom. 1997, 32, 277-286.

23. Nguyen, V. Q.; Turecek, F. J. Mass Spectrom. 1996, 31, 11731184.

24. Nguyen, V. Q.; Turecek, F. J. Mass Spectrom. 1997, 32, 55-63. 